\title{
Primary pericardial extragastrointestinal stromal tumor: A case report and literature review
}

\author{
TANER ARPACI ${ }^{1}$, FATMA TOKAT ${ }^{2}$, RABIA BOZDOGAN ARPACI $^{3}$, TUGANA AKBAS $^{1}$, \\ GAMZE UGURLUER $^{4}$ and SINAN YAVUZ ${ }^{5}$
}

\begin{abstract}
${ }^{1}$ Department of Radiology, Acibadem University, Acibadem Adana Hospital, Adana 01130; ${ }^{2}$ Department of Pathology, Acibadem Maslak Hospital, Istanbul 34398; ${ }^{3}$ Department of Pathology, Mersin University, Mersin 33000; Departments of ${ }^{4}$ Radiation Oncology and ${ }^{5}$ Oncology, Acibadem University, Acibadem Adana Hospital, Adana 01130, Turkey
\end{abstract}

Received June 4, 2014; Accepted March 4, 2015

DOI: $10.3892 / \mathrm{ol} .2015 .3126$

\begin{abstract}
Gastrointestinal stromal tumors (GISTs) are the most prevalent mesenchymal tumors of the gastrointestinal tract. GISTs are considered to originate from the interstitial cells of Cajal, the pacemakers of the peristaltic activity of the gastrointestinal tract. More than $95 \%$ of GISTs express KIT protein and discovered on GIST-1. GISTs may also be encountered in locations outside the gastrointestinal tract, in which case they are referred to as extra-GISTs (EGISTs) and often behave more aggressively. This is the case report of a primary pericardial EGIST in a 53-year-old male patient, confirmed by immunohistochemistry. To the best of our knowledge, this is the third case of EGIST diagnosed above the diaphragm, without being associated with the esophageal wall. Two cases of primary EGIST arising from the pleura were reported previously. In addition, this is the first reported case of an EGIST originating from the pericardium.
\end{abstract}

\section{Introduction}

Gastrointestinal stromal tumors (GISTs) are the most prevalent mesenchymal tumors of the gastrointestinal tract (1). GISTs are considered to originate from the interstitial cells of Cajal (ICC), which are the pacemakers of the peristaltic activity of the gastrointestinal tract. GISTs mainly emerge in the stomach $(60 \%)$, jejunum and ileum $(30 \%)$, colorectum, duodenum, esophagus and appendix (10\%). However, they may also be encountered in locations outside the gastrointestinal tract, such as the omentum, mesentery and retroperitoneum, in which case they are referred to extra-GISTs (EGISTs) (2). More than 95\% of GISTs express KIT protein and discovered on GIST-1 (DOG-1), which are considered to be the most specific and

Correspondence to: $\mathrm{Dr}$ Taner Arpaci, Department of Radiology, Acibadem University, Acibadem Adana Hospital, 66 Cumhuriyet Street, Adana 01130, Turkey

E-mail: tanerarpaci@yahoo.com

Key words: gastrointestinal stromal tumor, pericardium, computed tomography, immunohistochemistry sensitive diagnostic markers for GISTs. Approximately $80 \%$ of GISTs harbor a KIT mutation and 8-10\% express mutations in the platelet-derived growth factor receptor, $\alpha$ polypeptide (PDGFR $\alpha$ ). Imatinib, a selective inhibitor of KIT and PDGFR $\alpha$, is a treatment agent effective against EGISTs $(1,2)$.

\section{Case report}

A 53-year-old male patient with severe dyspnea was admitted to the Acibadem Adana Hospital (Adana, Turkey). A chest computed tomography (CT) scan revealed a massive pericardial effusion and pericardiocentesis was performed. A positron emission tomography (PET)/CT scan performed following recurrence of pericardial effusion revealed right pericardial hypermetabolic mass lesions [maximum standardized uptake value (SUV), 12.1], pericardial effusion, right hilar lymphadenopathy (maximum SUV, 4.9) and multiple metastatic nodules in both lungs. There was no evidence of a primary malignancy originating from the abdominopelvic cavity. The chest CT and coronary CT angiography revealed that the pericardial mass involved the right atrium and exhibited peripheral contrast enhancement. There was no association between the pericardial mass and esophagus (Fig. 1). The lesion was considered to be inoperable; a right thoracotomy and wedge resection were performed and a small number of lung nodules were resected. Microscopically, the tumor was composed of spindle or ovoid cells with eosinophilic cytoplasm and pleomorphic nuclei with conspicuous nucleoli. Mitotic figures were frequently observed. Immunohistochemically, the tumor cells were strongly positive for cluster of differentiation 117 (CD117; c-Kit) and CD34 and exhibited heterogeneous moderate positivity for DOG-1 (Fig. 2). The Ki-67 labeling index was $61 \%$. The pathological diagnosis was malignant EGIST. Therapy with adjuvant imatinib (400 mg/day) was recommended. At the beginning of treatment, the patient responded well, however, after a further 6 months the disease progressed and the patient succumbed to the disease 18 months later. To the best of our knowledge, this is the third reported case of EGIST occurring above the diaphragm without being associated with the esophageal wall. Two cases of primary EGIST originating from the pleura were reported previously $(1,2)$. In addition, this is the first reported case of an EGIST originating from the pericardium. 

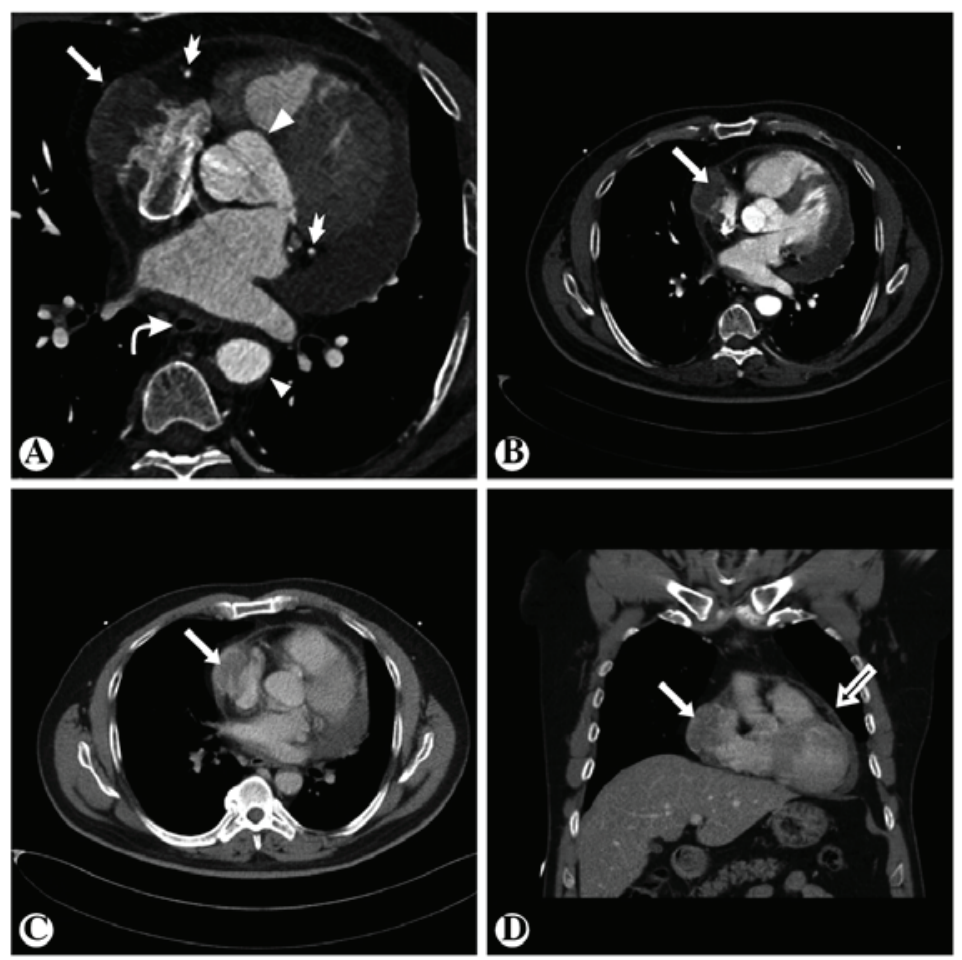

Figure 1. Images of (A and B) coronary CT angiography, (C) axial and (D) reformatted coronal chest CT, showing right pericardial rim-enhancing hypodense mass lesions (white arrows) involving the right atrium. The esophagus (curved arrow), aorta (arrowheads), coronary arteries (short arrows) and the remaining cardiac chambers are normal. Diffuse pericardial thickening and effusion are also present (black-on-white arrow). CT, computed tomography.
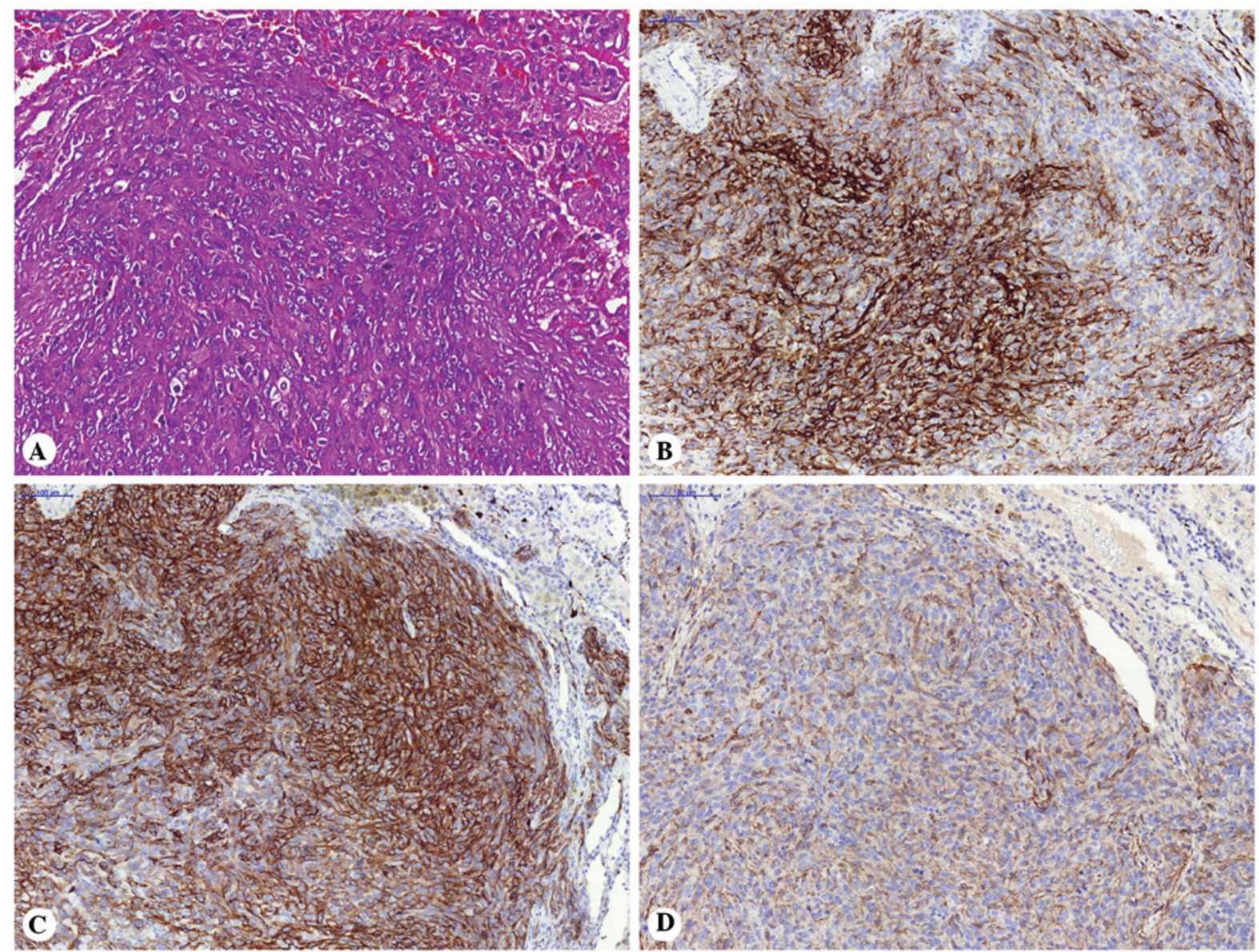

Figure 2. Immunohistochemical analysis of the resected tumor nodules. (A) The tumor was composed of spindle cells with eosinophilic cytoplasm (hematoxylin and eosin staining; magnification, x20). The tumor cells exhibited (B) strong diffuse cytoplasmic immunoreactivity for CD34; (C) intense cytoplasmic staining for CD117; and (D) moderate heterogeneous staining for discovered on gastrointestinal stromal tumor 1. CD, cluster of differentiation. 


\section{Discussion}

EGISTs are considered to originate from the common precursor cells that differentiate into this ICC-derived neoplasm during development outside of the gastrointestinal tract. Another theory is that this tumor may originate from the pluripotent mesenchymal stem cells located outside of the gastrointestinal tract (3). Contrary to GISTs, the clinical findings of EGISTs are not comprehensively known and are often associated with adverse prognostic factors, such as high proliferative index, large size, lymph node involvement and distant metastasis. The emergence of EGIST outside the gastrointestinal tract may lead to a delay in the occurrence of clinical symptoms. A number of these cases are diagnosed at a late stage, making surgical resection difficult $(1,2)$. A mutational study on EGISTs demonstrated that this type of neoplasm exhibits a lower frequency $(41.4 \%)$ of KIT mutation at exon 11, which may be the reason underlying the good response to imatinib (4). Radiologically, these tumors are often identified in the bowel wall, involving either the mucosal or serosal surface. The tumor size ranges between 1 and $35 \mathrm{~cm}$, with a median size of $5 \mathrm{~cm}$. In approximately two-thirds of the cases the tumor margins are well-defined. Large tumors, in particular, may display areas of hemorrhage and necrosis, leading to a heterogeneous appearance on imaging. The enhancement pattern may be homogeneous or heterogeneous (5-7).

\section{References}

1. Yi JH, Sim J, Park BB, et al: The primary extra-gastrointestinal stromal tumor of pleura: a case report and a literature review. Jpn J Clin Oncol 43: 1269-1272, 2013.

2. Long KB, Butrynski JE, Blank SD, et al: Primary extragastrointestinal stromal tumor of the pleura: report of a unique case with genetic confirmation. Am J Surg Pathol 34: 907-912, 2010.

3. Miettinen $\mathrm{M}$ and Lasota J: Gastrointestinal stromal tumors: pathology and prognosis at different sites. Semin Diagn Pathol 23: 70-83, 2006.

4. Yamamoto H, Oda Y, Kawaguchi K, et al: c-Kit and PDGFRA mutations in extragastrointestinal stromal tumor (gastrointestinal stromal tumor of the soft tissue). Am J Surg Pathol 28: 479-488, 2004.

5. Lee CM, Chen HC, Leung TK and Chen YY: Gastrointestinal stromal tumor: computed tomographic features. World J Gastroenterol 10: 2417-2418, 2004.

6. Levy AD, Remotti HE, Thompson WM, Sobin LH and Miettinen M: Gastrointestinal stromal tumors: radiologic features with pathologic correlation. Radiographics 23: 283-304, 456, quiz 532, 2003.

7. Ulusan S, Koc Z and Kayaselcuk F: Gastrointestinal stromal tumours: CT findings. Br J Radiol 81: 618-623, 2008. 